\title{
Differential interferometry using a Bose-Einstein condensate ${ }^{\star}$
}

\author{
Matthias Gersemann $^{1, a}$, Martina Gebbe ${ }^{2}$, Sven Abend ${ }^{1}$, Christian Schubert ${ }^{1,3}$, and Ernst M. Rasel ${ }^{1}$ \\ 1 Institut für Quantenoptik, Gottfried Wilhelm Leibniz Universität Hannover, Welfengarten 1, D-30167 Hannover, Germany \\ 2 Zentrum für angewandte Raumfahrttechnologie und Mikrogravitation (ZARM), Universität Bremen, Am Fallturm D-28359, \\ Bremen, Germany \\ 3 Deutsches Zentrum für Luft- und Raumfahrt e.V. (DLR), Institut für Satellitengeodäsie und Inertialsensorik, c/o Leibniz \\ Universität Hannover, DLR-SI, Callinstraße 36, 30167 Hannover, Germany
}

Received 28 July 2020 / Accepted 14 August 2020

Published online 1 October 2020

(C) The Author(s) 2020. This article is published with open access at Springerlink.com

\begin{abstract}
Out of a single Bose-Einstein condensate (BEC), we create two simultaneous interferometers, as employed for the differentiation between rotations and accelerations. Our method exploits the precise motion control of BECs combined with the precise momentum transfer by double Bragg diffraction for interferometry. In this way, the scheme avoids the complexity of two BEC sources and can be readily extended to a six-axis quantum inertial measurement unit.
\end{abstract}

\section{Introduction}

Atom interferometers have various applications in many different fields like fundamental physics $[1-6]$, geodesy [7-10], and inertial sensing [11]. Exploiting cold or ultracold atomic ensembles, they serve to precisely measure rotations [12-15], accelerations $[10,16,17]$, and gravity gradients $[18,19]$. With few exceptions, most experiments today use laser-cooled atoms. Their dominating systematic errors are connected to the motion of the atoms $[10$, $20,21]$. The latter can be reduced by using ensembles with a narrower momentum distribution and a well-controlled mean velocity.

In this paper, we demonstrate a dual BEC interferometer sensitive to rotations and accelerations. Indeed, presently the most narrow momentum distributions are achieved by exploiting evaporated atoms or even BECs [22-24]. The application of BECs has several advantages: (i) their small spatial wave packet extension and expansion reduce effects stemming from the coherent interaction with inhomogeneous light fields [21,25] creating the interferometer. (ii) They allow extending the time the atoms spend in the interferometer. (iii) Moreover, BECs enable efficient Bragg and Raman processes and, therefore, a high interferometric contrast in the corresponding interferometers $[26,27]$.

These features are especially beneficial for inertial sensing with so-called Mach-Zehnder-type atom interferometers (MZI) where a wave packet is subsequently split,

\footnotetext{
* Contribution to the Topical Issue "Quantum Technologies for Gravitational Physics", edited by Tanja Mehlstäubler, Yanbei Chen, Guglielmo M. Tino, Hsien-Chi Yeh.

a e-mail: gersemann@iqo.uni-hannover.de
}

reflected, and recombined by interacting with three successive, retro-reflected laser pulses driving Bragg or Raman processes [28]. At each process $j=$ I, II or III, a phase $\phi_{j}$ is imprinted, giving rise to the so-called laser phase $\phi_{\mathrm{L}}=\phi_{\mathrm{I}}-2 \phi_{\mathrm{II}}+\phi_{\mathrm{III}}[29]$. In leading order, the total phase shift $\phi$ of these interferometers depends, apart from the laser phase $\phi_{\mathrm{L}}$, via two contributions $\phi_{\mathrm{a}}$ and $\phi_{\mathrm{r}}$ on the motion of the atoms with respect to the retro-reflector [30]. The first one stems from accelerations $\boldsymbol{a}$ according to

$$
\phi_{\mathrm{a}}=\boldsymbol{k}_{\mathrm{eff}} \cdot \boldsymbol{a} T^{2},
$$

where $\boldsymbol{k}_{\text {eff }}=2 n \boldsymbol{k}$ defines the momentum transferred to the atoms due to an $n$-th order Bragg or Raman process, $\boldsymbol{k}$ the corresponding wave vector and $T$ the time between the light pulses. The second contribution comes from rotations $\boldsymbol{\Omega}$, and arises when the interferometer encloses an area due to a non-vanishing mean velocity $\boldsymbol{v}$ of the atoms giving rise to the atomic Sagnac effect [31]

$$
\phi_{\mathrm{r}}(\boldsymbol{v})=2 \boldsymbol{k}_{\mathrm{eff}} \cdot(\boldsymbol{\Omega} \times \boldsymbol{v}) T^{2} .
$$

These equations underline the importance of precise control of the momentum transfer $\boldsymbol{k}_{\text {eff }}$ in the interferometer and the mean velocity $\boldsymbol{v}$. In contrast to the acceleration phase term, the rotation phase term also depends on the sign of $\boldsymbol{v}$. Hence, two atom interferometers operating with wave packets travelling with opposite forward velocity $\boldsymbol{v}$ can be exploited to differentiate between accelerations and rotations by adding or subtracting the half sum of the total interferometer phase shift $\phi_{i}=\phi_{a, i}+\phi_{r, i}(\boldsymbol{v})+\phi_{\mathrm{L}, i}$ as follows

$$
\begin{aligned}
\phi_{\mathrm{a}} & =\frac{1}{2}\left(\phi_{1}(\boldsymbol{v})+\phi_{2}(-\boldsymbol{v})\right), \text { and } \\
\phi_{\mathrm{r}} & =\frac{1}{2}\left(\phi_{1}(\boldsymbol{v})-\phi_{2}(-\boldsymbol{v})\right),
\end{aligned}
$$


where $i=1,2$ denotes the two interferometers and the laser phase $\phi_{\mathrm{L}}$ is assumed to be equal for both interferometers. This approach was demonstrated in the seminal work of a gyroscope based on a dual atomic beam [32] and was later extended to two sources delivering laser-cooled atoms $[13,14]$.

In our approach, we employ first-order double Bragg diffraction to create two wave packets from a single BEC, which separate from each other in opposite direction according to the transferred momentum of four photon recoils with a relative velocity $\boldsymbol{v}_{\text {sep }}$. This generates two sources for two interferometers from a single atomic ensemble [18,33-35]. Therefore it avoids issues connected to multiple sources, such as uncertainties in initial velocities and positions causing systematic errors in the differential signal of the two interferometers $[13,14,36]$. The two wave packets serve as the input for two simultaneously operated Mach-Zehnder interferometers and their individual velocities $\pm \boldsymbol{v}_{\text {sep }} / 2$ are orders of magnitude larger than their expansion rate, such that they can be well distinguished even after a short time of flight.

In our case, the half sum of the measured phases evaluates to

$$
\phi_{\mathrm{a}}=\boldsymbol{k}_{\mathrm{eff}} \cdot \boldsymbol{a} T^{2}+\phi_{\mathrm{L}}
$$

and the half difference to

$$
\phi_{\mathrm{r}}=2 \boldsymbol{k}_{\mathrm{eff}} \cdot\left(\boldsymbol{\Omega} \times \frac{\boldsymbol{v}_{\mathrm{sep}}}{2}\right) T^{2} .
$$

To first order, the differential phase $\phi_{\mathrm{r}}$ is only dependent on the well controllable vector $\boldsymbol{v}_{\text {sep }}$, generated by the double Bragg process, and independent of any initial velocity $\boldsymbol{v}_{\mathbf{0}}$. For the sum phase, spurious contributions associated with a non-zero initial velocity can be reduced by the well-controlled release of the BEC, i.e. to less than $\pm v_{0}=60 \mu \mathrm{m} / \mathrm{s}$ per shot $[37,38]$ and are therefore neglected here. Equations (5) and (6) also imply the suppression of rotation noise in $\phi_{\mathrm{a}}$ and of acceleration noise in $\phi_{\mathrm{r}}$.

\section{Dual BEC interferometer}

The experimental scheme of our dual BEC interferometer is illustrated in Figure 1a. Initially, a single BEC is created on an atom chip as detailed in [39]. The setup produces ensembles of up to $1.5 \times 10^{4}{ }^{87} \mathrm{Rb}$ atoms within $15 \mathrm{~s}$ in the hyperfine state $F=2, m_{F}=2$ at around $50 \mathrm{nK}$ effective temperature. After creation, the BEC is released from the trap and transferred via an adiabatic rapid passage to the magnetic state $m_{F}=0$. The ensemble is symmetrically split employing first-order double Bragg diffraction in a twin lattice, i.e. two counter-propagating optical lattices, as described in [40], with a diffraction efficiency in the order of $95 \%$, limited by spurious thermal background atoms and spontaneous emission. In this way, two wave packets are created that travel with a relative velocity $v_{\text {sep }}=4 \hbar k / m$ apart from each other along the $y$-axis, where $m$ denotes the atomic mass and $\hbar$ the (a)

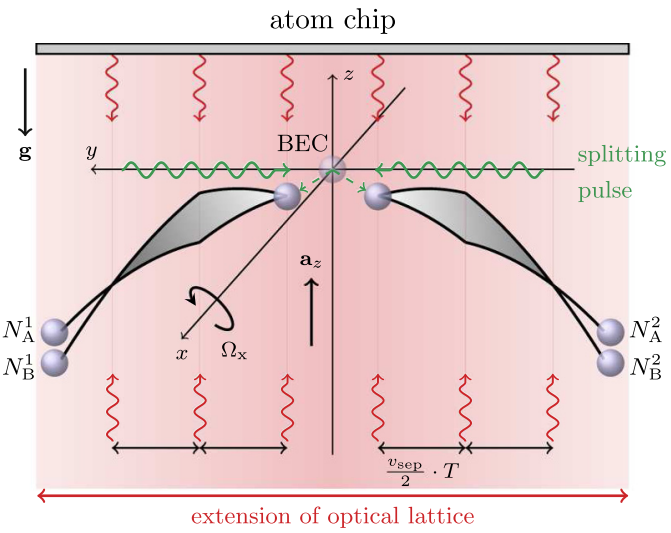

(b)
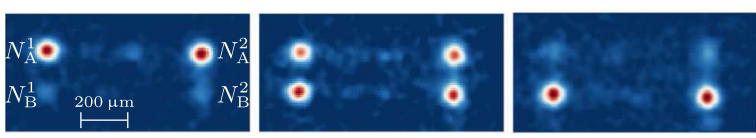

Fig. 1. Dual BEC interferometer. (a) A BEC released in free fall (gravity $\boldsymbol{g}$ aligned parallel to the $z$-axis) is split via double Bragg diffraction (green arrows) into two wave packets separating in $y$-direction with $v_{\text {sep }}=4 \hbar k / m$ apart from each other. Two Mach-Zehnder-type interferometers are formed by three successive light pulses (red arrows), separated by intervals of $T$. The light pulses induce Bragg diffraction in an optical lattice (red shaded area) retro-reflected from the atom chip at the top. Both interferometers are sensitive to accelerations $\boldsymbol{a}_{z}$ and rotations $\boldsymbol{\Omega}_{x}$. The atomic densities at the output ports of the interferometers are detected via imaging the absorption of light propagating in $x$-direction and exploited to determine the number of atoms in the ports. (b) Three exemplary absorption images corresponding to different phases $\phi_{\mathrm{L}}$ and, therefore, varying populations $P\left(\phi_{i}\right)$ in the output ports of the individual interferometers. The identical relative population for both interferometers shows the correlation of their phases $\phi_{1,2}$.

reduced Planck constant. Two Mach-Zehnder interferometers are formed by three successive first-order Bragg processes, separated by the interval $T$ and induced by illuminating the BEC with an optical lattice traveling in $z$-direction along the direction of gravity. The lattice is created by retro-reflecting a Gaussian-shaped light beam from the surface of the atom chip. The output atomic density distributions of the two interferometers are detected via absorption imaging with a CCD camera, as illustrated in Figure 1b for three exemplary phases $\phi_{\mathrm{L}}$.

We operate the dual BEC interferometer with $T=5 \mathrm{~ms}$, and record interferograms by varying $\phi_{\mathrm{L}}$, which determines the position of nodes and anti-nodes of the lattice driving the Bragg processes. In the absence of forces, a stepwise increase of $\phi_{\mathrm{L}}$ typically leads to a sinusoidal fringe pattern. In our experiment, however, vibrational noise randomly changes the phase of the two interferometers $\phi_{1,2}$ for each measurement and, hence, the sinusoidal dependence disappears. Modulo $2 \pi$, the output phase $\phi_{i}$ can be extracted from the normalized output population with

$$
P\left(\phi_{i}\right)=P_{0}-A \cdot \cos \left(\phi_{i}\right)=\frac{N_{\mathrm{A}}^{i}}{N_{\mathrm{A}}^{i}+N_{\mathrm{B}}^{i}},
$$




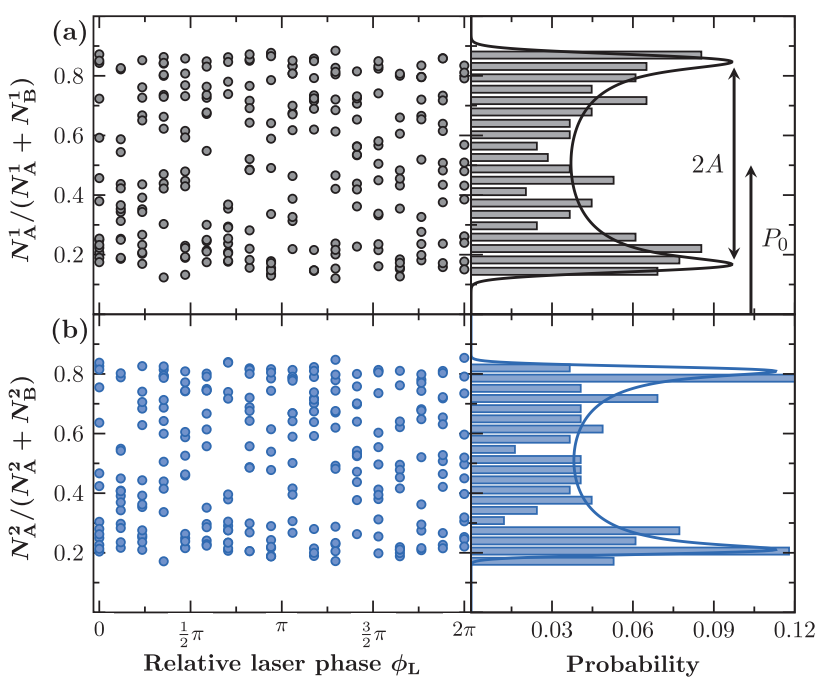

Fig. 2. Output signals $P\left(\phi_{i}\right)$ of the two Mach-Zehnder interferometers from Figure 1a and the corresponding histograms. The relative laser phase $\phi_{\mathrm{L}}$ is increased in steps of $2 \pi / 14$ between 0 and $2 \pi$. Vibrational noise washes out the expected sinusoidal response (left-hand side). A histogram analysis (right-hand side) reveals a characteristic double peak structure reflecting the sinusoidal dependence of the interference signal on $\phi_{\mathrm{L}}$. The contrast $C=A / P_{0}$, given by the amplitude $A$ of the signal divided by its mean $P_{0}$, is extracted from a kernel density estimation fitting routine of the distribution [43].

where $N_{\mathrm{A}}^{i}$ and $N_{\mathrm{B}}^{i}$ describe the atom numbers in the two output ports of the $i$-th interferometer (Fig. 1b).

\section{Analysis of the interferometer output}

Due to the present phase noise, we perform a statistical analysis in the form of histograms of the output port population [41] and calculate an Allan deviation [42] of the differential phase $\phi_{\mathrm{r}}$ to characterize the contrast and technical noise of our interferometer. Figure 2 shows the scattered populations $P\left(\phi_{i}\right)$ of the two interferometers for a step-wise increase of $\phi_{\mathrm{L}}$ between 0 and $2 \pi$ and the corresponding histogram representations. For both data sets, a characteristic double-peak structure appears, as seen in Figure 2 on the right-hand side. A kernel density estimation is applied to extract the amplitude $A$ of the signal, which scales with the efficiency of the Bragg processes [43], and its mean $P_{0}$. This allows us to determine the individual contrast values $C=A / P_{0}$. The evaluation yields the same value $P_{0}$ for the two interferometers but two different amplitudes $A$, resulting in a contrast of $C_{1}=0.67$ and $C_{2}=0.59$, respectively. For comparison, a single MZI in our setup with a pulse separation time of $T=0 \mathrm{~ms}$ features a contrast of $C=0.84$ for optimized pulse amplitudes. In this case, the contrast is limited by imperfect preparation of the atoms into $m_{F} \neq 0$ sub-states as well as by spontaneous emission occurring during the Bragg processes and spurious thermal background atoms.

The final separation of the wave packets in $y$-direction of about $600 \mu \mathrm{m}$ is relatively small compared to the $6.6 \mathrm{~mm}$

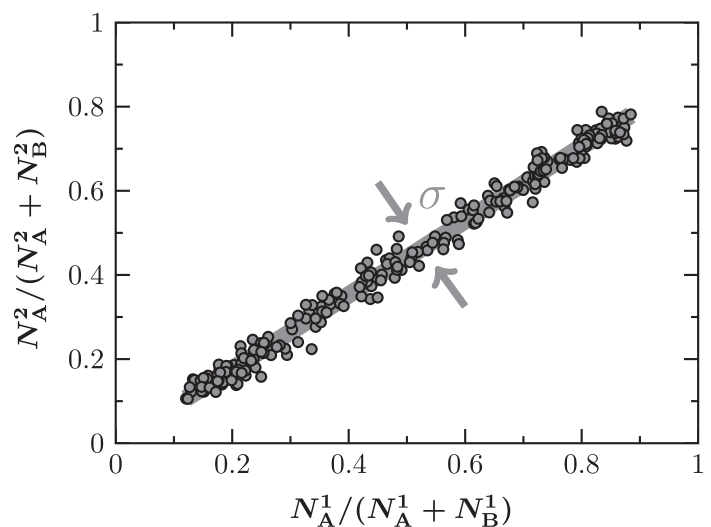

Fig. 3. Parametric plot of the normalised output signals $P\left(\phi_{i}\right)$ in Figure 2 of the two interferometers illustrated in Figure 1a. The formation of a line indicates a correlation without a significant differential phase shift. The spread of the data points along this line is determined by the residual noise $\sigma$ on the differential signal $\phi_{\mathrm{r}}$. This residual noise is much smaller than the noise on the individual interferometers.

diameter of the Gaussian interferometry beam. Consequently, we expect only minor variations of the diffraction efficiency due to the different positions of the wave packets within the Gaussian beam profile, and rather attribute the observed difference to imperfections of the retro-reflection coating of the atom chip for the optical lattice, causing intensity fluctuations across the beam.

The relative phase of both interferometers can be visualized by a parametric plot of their signals. This results in a so-called Lissajous curve which, in principle, can be exploited for ellipse-specific fitting methods [44]. The linear dependence appearing in our data set (Fig. 3) indicates that the random phase jitter is mostly common to both interferometers, implying a negligible phase shift (modulo $2 \pi)$ and low noise in the differential signal $\phi_{\mathrm{r}}$ when compared to the sum signal $\phi_{\mathrm{a}}$. The standard deviation of a linear fit, along with the mean contrast, can be related to a differential phase noise value, which may include residual inertial phase noise, but also non-inertial noise sources like amplitude and detection noise. This evaluation yields a value of $\sigma=16.2 \mathrm{mrad}$, and implicitly assumes the absence of a mean differential phase.

For a more thorough analysis, we calculate the phases for each interferometer time series separately by solving equation (7) for $\phi_{i}$, inserting the normalized population $P$, offset $P_{0}$, and amplitude $A$, as determined from Figure 2 . These $\phi_{i}$ are employed to calculate the corresponding half difference data set $\phi_{\mathrm{r}}$ following equation (4). The Allan deviation of $\phi_{\mathrm{r}}$ (Fig. 4) allows to assess the temporal characteristics of the noise. This deviation yields a single-shot phase noise equivalent of $\sigma_{\phi_{\mathrm{r}}}=16.4 \pm 2 \mathrm{mrad}$ matching our estimation from the parametric plot within the error bars. We, therefore, deduce that the differential interferometery suppresses contributions from vibrational accelerations and the laser phase by at least $22 \mathrm{~dB}$. With these noise estimations and equation (6) the sensitivity to rotations can be estimated to $1.7 \times 10^{-3} \mathrm{rad} / \mathrm{s}$ per shot. After $1000 \mathrm{~s}$ of integration time, the Allan deviation yields a 


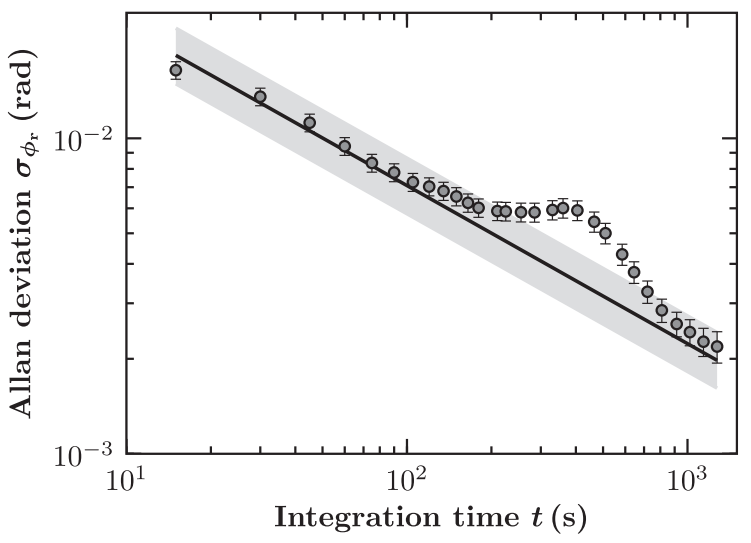

Fig. 4. Allan deviation of the differential phase $\phi_{\mathrm{r}}$ of our dual BEC interferometer with a measurement cycle time of $15 \mathrm{~s}$. The resulting starting value of $\sigma_{\phi_{\mathrm{r}}}=16.4 \pm 2 \mathrm{mrad}$ is estimating the noise per experimental cycle. The solid line depicts an estimation of the quantum projection noise, with the shaded area defining an uncertainty band due to the atom number determination in the experiment. Apart from a peak at $400 \mathrm{~s}$, which matches the modulation frequency of the air condition, the functional dependence of $1 / \sqrt{t}$ agrees well with signal averaging in the presence of white noise.

value of $\sigma_{\phi_{\mathrm{r}}}=2.0 \pm 1 \mathrm{mrad}$ corresponding to a sensitivity of $2 \times 10^{-4} \mathrm{rad} / \mathrm{s}^{1}{ }^{1}$ The noise in the differential signal agrees with the estimated quantum projection noise as depicted in Figure 4 (solid line) and given by

$$
\sigma_{\mathrm{qpn}}, \phi_{\mathrm{r}}=\frac{1}{2} \sqrt{\frac{1}{\left(C_{1} \sqrt{N_{\mathrm{A}}^{1}+N_{\mathrm{B}}^{1}}\right)^{2}}+\frac{1}{\left(C_{2} \sqrt{N_{\mathrm{A}}^{2}+N_{\mathrm{B}}^{2}}\right)^{2}}},
$$

which is an upper bound for the sensitivity of our measurement. The uncertainty in determining the atom number in our experiment is estimated to $\pm 50 \%$, which leads to an uncertainty for $\sigma_{\mathrm{qpn}, \phi_{\mathrm{r}}}$ depicted in the form of the shaded areas around the solid line.

\section{Multi-axis operation}

In our current setup, two wave packets are generated, separating along the $y$-axis and serving as input for two MZIs, to measure the rotation component $\boldsymbol{\Omega}_{x}$ and the acceleration component $\boldsymbol{a}_{z}$, as shown in Figure 1a. Employing three perpendicular oriented optical lattices, as depicted in Figure 5a, this scheme can be extended to a quantum inertial measurement unit $[35,45,46]$, successively detecting all three components of $\boldsymbol{\Omega}$ and $\boldsymbol{a}$ by performing correlated interferometry. For all measurements, two wave packets with a relative velocity $\boldsymbol{v}_{\text {sep }}$ are generated by double Bragg diffraction. Afterwards, an optical lattice drives single or double Bragg processes in the direction perpendicular to the respective $\boldsymbol{v}_{\text {sep }}$. In the directions perpendicular to gravity, a retro-reflected light beam featuring two frequencies induces double diffraction, as shown in

\footnotetext{
1 Roughly a factor of 3 above the angular speed of the earth.
}

Figures $1 \mathrm{a}, 5 \mathrm{~b}$ and $5 \mathrm{c}$ along the $x$ - and $y$-axis. Along gravity, the lattice has to be accelerated to drive single Bragg diffraction and double diffraction requires an additional light field with a third frequency component [47]. Alternatively, for the initial generation of the two wave packets along this direction, as needed for the case in Figure 5b, double diffraction can also be implemented right after the release of the BEC or with a fountain sequence where the condensate is launched to the point of nearly zero velocity [22].

In contrast to simultaneous measurements [35], successive operation imposes fewer demands on laser power to coherently manipulate the atoms as well as on efforts to suppress losses due to cross-talk of the Bragg processes along the different axes.

Imaging the population of the individual output ports can be performed off-axis with a single CCD camera for all three geometries. To avoid the requirement of additional optical access, illumination of the atoms for fluorescence detection can be performed along the existing optical access used for the lattices. This in total allows for a compact setup and extends earlier approaches based on laser-cooled sources [11] to precisely controllable BECs without compromises in complexity.

\section{Applications in inertial sensing}

Our scheme offers interesting prospects for inertial navigation and the measurement of the Earth's rotational components. For both applications, the sensitivity of the dual BEC interferometer towards inertial forces can be increased by either enlarging the pulse separation time $T$, the momentum transfer $k_{\text {eff }}$, or, in case of rotations, the separation velocity $v_{\text {sep }}$, as apparent from equations ((5) and (6)). Larger momentum transfer can be realized with successive first- or higher-order transitions [48-52] or in combination with Bloch oscillations [53] in the form of a twin lattice [54]. Higher velocities $v_{\text {sep }}$ and longer times $T$, however, require optical lattices with larger waists, and, thus more laser power. Compared to Gaussian beams, flattop shaped profiles show the advantageous feature of a more equal and efficient distribution of laser power over the interferometry region [55], which benefits the scheme presented here.

Table 1 shows the sensitivities for our current setup and two scenarios for future applications. The lattice beam diameter $d \geq v_{\text {sep }} \cdot 2 T$ determines the maximum spacetime area of the interferometer, as illustrated by the red shaded area in Figure 1 and the individual optical lattices in Figure 5a. This sets a limit on the product of velocity $v_{\text {sep }}$ and time $T$, under the assumption that the full time the two wave packets spent in the lattice beam can be used for the interferometer and that the initial ensemble is located in the center of this beam. Higher velocities $v_{\text {sep }}$ reduce the available time $T$ and, therefore, allow higher repetition rates. Larger times $T$, on the other hand, increase the acceleration and rotation sensitivity quadratically.

For a quantum inertial measurement unit we consider a flat-top beam diameter of $d=15 \mathrm{~mm}$, allowing for a 

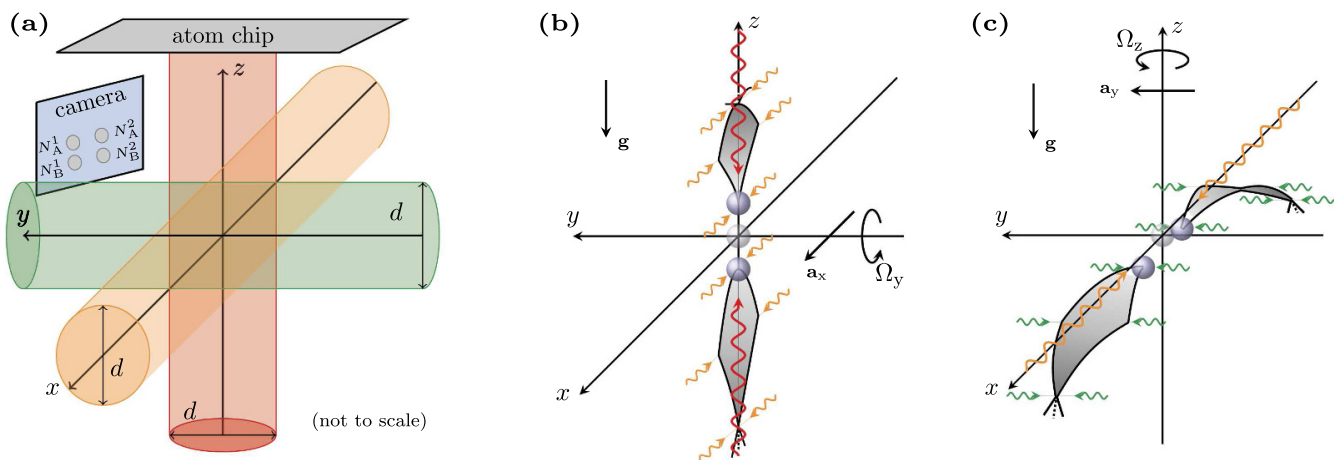

Fig. 5. Setup to measure all components of $\boldsymbol{\Omega}$ and $\boldsymbol{a}$. (a) In extension to Figure 1a, three perpendicular oriented optical lattices in red ( $z$-axis), green ( $y$-axis) and orange $(x$-axis) are needed for the various diffraction processes. Off-axis imaging of the atomic ensembles is shown exemplary for the geometry in Figure 1a. In order to measure $\boldsymbol{\Omega}_{y}$ and $\boldsymbol{\Omega}_{z}$, as well as $\boldsymbol{a}_{\boldsymbol{x}}$ and $\boldsymbol{a}_{\boldsymbol{y}}$, we change the direction of the double Bragg process to generate two initial wave packets along the $z$-axis (b) or the $x$-axis (c). The corresponding interferometers are formed by three successive light pulses inducing double diffraction along the $x$ - and $y$-axis respectively.

Table 1. Sensitivity limits of the dual BEC interferometer per shot based on the parameters atom number $N$, pulse separation time $T$, contrast $C$, separation velocity $v_{\text {sep }}$, and momentum $\hbar k_{\text {eff }}$ transferred within the Mach-Zehnder sequence. The parameter sets are constrained by the achievable space-time area of the interferometer, limited by the interferometry beam diameter $d$. Next to our work, the parameters for two applications, one for navigation purposes and one for Earth rotation sensing are presented and the related sensitivities per cycle are given.

\begin{tabular}{lcccccccc}
\hline & $\begin{array}{c}\text { Beam diameter } \\
d[\mathrm{~mm}]\end{array}$ & $N$ & $T[\mathrm{~ms}]$ & $C$ & $\begin{array}{c}v_{\text {sep }} \\
{[\hbar k / m]}\end{array}$ & $\begin{array}{c}\hbar k_{\text {eff }} \\
{[\hbar k]}\end{array}$ & $\begin{array}{c}\text { Acceleration sensitivity } \\
\text { limit }\left[\mathrm{m} / \mathrm{s}^{2}\right] 1 /\left(\sigma_{\mathrm{qpn}} k_{\mathrm{eff}} T^{2}\right)\end{array}$ & $\left.\begin{array}{c}\text { Rotation sensitivity } \\
\text { limit }[\mathrm{rad} / \mathrm{s}]\end{array}\right] /\left(\sigma_{\mathrm{qpn}} k_{\mathrm{eff}} v_{\mathrm{sep}} T^{2}\right)$ \\
\hline Current setup & $6.6^{a}$ & $1.5 \times 10^{4}$ & 5 & 0.64 & 4 & 2 & $3.1 \times 10^{-5}$ & $1.3 \times 10^{-3}$ \\
Navigation & $15^{b}$ & $1 \times 10^{5}$ & 25 & 0.50 & 32 & 16 & $7.9 \times 10^{-8}$ & $4.2 \times 10^{-7}$ \\
Earth monitoring & $50^{b}$ & $1 \times 10^{6}$ & 125 & 0.50 & 32 & 96 & $1.7 \times 10^{-10}$ & $8.8 \times 10^{-10}$ \\
\hline
\end{tabular}

${ }^{a}$ Gaussian beam diameter $2 w_{0},{ }^{b}$ flat-top beam diameter

fairly compact setup. The parameter set of $v_{\text {sep }}=32 \hbar k$ and $T=25 \mathrm{~ms}$ is chosen in a way that the diameter $d$ roughly equals the (vertical) baseline of the interferometer $\left(\frac{1}{2} g(2 T)^{2} \approx 12 \mathrm{~mm}\right)$ if the geometry is aligned as shown in Figure 1. For a contrast of $C=0.5$, as reported in [54] and a total atomic flux of $N=1 \times 10^{5}$ per $1.6 \mathrm{~s}$ demonstrated in [56] an acceleration sensitivity on the order of $10^{-8} \mathrm{~m} / \mathrm{s}^{2}$ and a rotation sensitivity of $10^{-7} \mathrm{rad} / \mathrm{s}$ can be achieved at a cycle time of less than two seconds.

High-resolution Earth rotation measurements as performed by large ring laser structures [57,58], require much higher sensitivities and, therefore, typically larger setups. In contrast to the previous application, we assume here a flat-top laser beam diameter of $d=50 \mathrm{~mm}$, an extended pulse separation time $T=125 \mathrm{~ms}$, an improved atom number of $N=1 \times 10^{6}$ and an increased momentum transfer of $\hbar k_{\text {eff }}=96 \hbar k$ in the interferometer. For this estimation a suitable laser power of $10 \mathrm{~W}$ would correspond to a constant lattice depth of around $V_{0}=9 E_{\mathrm{r}}$, in units of recoil energy, when $90 \%$ of the total power is equally distributed over the flat-top beam diameter. In this way, acceleration sensitivities of the order of $10^{-10} \mathrm{~m} / \mathrm{s}^{2}$ and rotation sensitivities of $10^{-10} \mathrm{rad} / \mathrm{s}$ seem feasible with our scheme.

\section{Conclusion}

In conclusion, we have presented a novel way of realizing a dual BEC interferometer exploiting double Bragg diffraction. Out of a single BEC in free fall we create two atomic wave packets traveling in opposite direction with a precise relative motion. Our approach combines the advantages of the excellent control BECs offer with a differential interferometric measurement without increasing the complexity associated with multiple atomic sources. It allows to perform rotation measurements in the differential phase while the sum phase is sensitive to accelerations. The measurement axes are determined by the light field alignment and the BEC release velocity, which can both be well controlled. Moreover, employing double Bragg diffraction does not heat and thus preserves the velocity distribution of the released and diffracted BEC.

With our concept, we show a route to the development of compact quantum sensors with combined acceleration and rotation sensitivity. Furthermore, it is straight forward to extend our scheme to a six-axis sensor employing three perpendicular laser beams and three sequential measurements, without an increase in size or laser power nor a reduction of the single-shot sensitivity of the atom interferometer. The simplicity of this scheme makes it suitable for the application in a quantum or hybrid inertial measurement unit [35] and applicable to on-board deployment on aircraft or ships [9]. In a larger-scale device, our scheme reaches sensitivities on the $10^{-10} \mathrm{rad} / \mathrm{s}$ level, able to precisely monitor the Earth's rotation and adds the feature of an alternative method of vibration suppression compared to butterfly-type atom interferometers [15]. The 
presented scheme is intrinsically symmetric and therefore directly applicable in a microgravity environment [59-61] to monitor rotation rates with suppressed environmental acceleration noise, thus enabling high precision measurements. Overall, it highlights the applicability and versatility of BECs for inertial measurements under a variety of different conditions.

Open access funding enabled and organized by Projekt DEAL. The presented work is supported by the CRC 1227 DQmat within the projects B07 and B09, the EXC 2123 Quantum Frontiers within the research units B02 and B05, the QUESTLFS, the German Space Agency (DLR) with funds provided by the Federal Ministry of Economic Affairs and Energy (BMWi) due to an enactment of the German Bundestag under grant No. DLR 50WM1952 (QUANTUS-V-Fallturm), 50WP1700 (BECCAL), 50RK1957 (QGYRO), the Verein Deutscher Ingenieure (VDI) with funds provided by the Federal Ministry of Education and Research (BMBF) under grant No. VDI 13N14838 (TAIOL), and received support from the CRC 1128 geo-Q within the projects A01 and A02. We acknowledge financial support from "Niedersächsisches Vorab" through the "Quantum- and Nano-Metrology (QUANOMET)" initiative within the project QT3 and through "Förderung von Wissenschaft und Technik in Forschung und Lehre" for the initial funding of research in the new DLR-SI Institute.

\section{Author contribution statement}

Sven Abend, Christian Schubert and Ernst M. Rasel developed the idea. Matthias Gersemann, Martina Gebbe and Sven Abend implemented the experiment, performed the measurements, derived the models and analysed the data. Christian Schubert verified the analytical methods and aided in interpreting the results. Ernst M. Rasel supervised the project and the findings of this work. All authors discussed the results and contributed to the manuscript. All authors have read and approved the final manuscript.

Publisher's Note The EPJ Publishers remain neutral with regard to jurisdictional claims in published maps and institutional affiliations.

Open Access This is an open access article distributed under the terms of the Creative Commons Attribution License (https://creativecommons.org/licenses/by/4.0/), which permits unrestricted use, distribution, and reproduction in any medium, provided the original work is properly cited.

\section{References}

1. G. Rosi, F. Sorrentino, L. Cacciapuoti, M. Prevedelli, G.M. Tino, Nature 510, 518 (2014)

2. P. Asenbaum, C. Overstreet, M. Kim, J. Curti, M.A. Kasevich, arXiv:2005.11624 (2020)

3. D. Schlippert, J. Hartwig, H. Albers, L.L. Richardson, C. Schubert, A. Roura, W.P. Schleich, W. Ertmer, E.M. Rasel, Phys. Rev. Lett. 112, 203002 (2014)
4. L. Zhou, S. Long, B. Tang, X. Chen et al., Phys. Rev. Lett. 115, 013004 (2015)

5. H. Parker, C. Yu, W. Zhong, B. Estey, H. Müller, Science 360, 6385 (2018)

6. R. Bouchendira, P. Cladé, S. Guellati-Khélifa, F. Nez, F. Biraben, Phys. Rev. Lett. 106, 080801 (2011)

7. V. Ménoret, P. Vermeulen, N. Le Moigne, S. Bonvalot, P. Bouyer, A. Landragin, B. Desruelle, Sci. Rep. 8, 12300 (2018)

8. X. Wu, Z. Pagel, B.S. Malek, T.H. Nguyen, F. Zi, D.S. Scheirer, H. Müller, Sci. Adv. 5, 9 (2019)

9. Y. Bidel, N. Zahzam, C. Blanchard, A. Bonnin, M. Cadoret, A. Bresson, D. Rouxel, M.-F. Lalancette, Nat. Commun. 9, 1 (2018)

10. C. Freier, M. Hauth, V. Schkolnik, B. Leykauf, M. Schilling, H. Wziontek, H-G. Scherneck, J. Müller, A. Peters, J. Phys.: Conf. Ser 723, 012050 (2016)

11. B. Canuel, F. Leduc, D. Holleville, A. Gauguet, J. Fils, A. Virdis, A. Clairon, N. Dimarcq, C.J. Bordé, A. Landragin, P. Bouyer, Phys. Rev. Lett. 97, 010402 (2006)

12. J.K. Stockton, K. Takase, M.A. Kasevich, Phys. Rev. Lett. 107, 133001 (2011)

13. A. Gauguet, B. Canuel, T. Léveque, W. Chaibi, A. Landragin, Phys. Rev. A 80, 063604 (2009)

14. P. Berg, S. Abend, G. Tackmann, C. Schubert, E. Giese, W.P. Schleich, F.A. Narducci, W. Ertmer, E.M. Rasel, Phys. Rev. Lett. 114, 063002 (2015)

15. D. Savoie, M. Altorio, B. Fang, L.A. Sidorenkov, R. Geiger, A. Landragin, Sci. Adv. 4, 12 (2018)

16. A. Peters, K.Y. Chung, S. Chu, Nature 400, 849 (1999)

17. P. Gillot, O. Francis, A. Landragin, F. Pereira Dos Santos, S. Merlet, Metrologia 51, L15 (2014)

18. P. Asenbaum, C. Overstreet, T. Kovachy, D.D. Brown, J.M. Hogan, M.A. Kasevich, Phys. Rev. Lett. 118, 183602 (2017)

19. J.M. McGuirk, G.T. Foster, J.B. Fixler, M.J. Snadden, M.A. Kasevich, Phys. Rev. A 65, 033608 (2002)

20. P. Gillot, B. Cheng, A. Imanaliev, S. Merlet, F. Pereira Dos Santos, The LNE-SYRTE cold atom gravimeter, in 2016 European Time and Frequency Forum (EFTF) (York, 2016), p. 1

21. A. Louchet-Chauvet, T. Farah, Q. Bodart, A. Clairon, A. Landragin, S. Merlet, F. Pereira Dos Santos, New J. Phys. 13, 065025 (2011)

22. S. Abend, M. Gebbe, M. Gersemann, H. Ahlers, H. Müntinga, E. Giese, N. Gaaloul, C. Schubert, C. Lämmerzahl, W. Ertmer, W.P. Schleich, E.M. Rasel, Phys. Rev. Lett. 117, 203003 (2016)

23. H. Müntinga, H. Ahlers, M. Krutzik, A. Wenzlawski, S. Arnold, D. Becker, K. Bongs, H. Dittus, H. Duncker, N. Gaaloul et al., Phys. Rev. Lett. 110, 093602 (2013)

24. T. Kovachy, J.M. Hogan, A. Sugarbaker, S.M. Dickerson, C. A. Donnelly, C. Overstreet, M.A. Kasevich, Phys. Rev. Lett. 114, 143004 (2015)

25. V. Schkolnik, B. Leykauf, M. Hauth, C. Freier, A. Peters, Appl. Phys. B 120, 311 (2015)

26. S.S. Szigeti, J.E. Debs, J.J. Hope, N.P. Robins, J.D. Close, New J. Phys. 14, 023009 (2012)

27. S. Hartmann, J. Jenewein, E. Giese, S. Abend, A. Roura, E.M. Rasel, W.P. Schleich, Phys. Rev. A 101, 053610 (2020)

28. M.A. Kasevich, S. Chu, Phys. Rev. Lett. 67, 181 (1991)

29. C.J. Bordé, Gen. Relat. Gravit. 36, 475 (2004) 
30. K. Bongs, R. Launay, M.A. Kasevich, Appl. Phys. B 84, 599 (2006)

31. F. Riehle, Th. Kisters, A. Witte, J. Helmcke, C.J. Bordé, Phys. Rev. Lett. 67, 177 (1991)

32. T.L. Gustavson, A. Landragin, M.A. Kasevich, Class. Quantum Gravity 17, 2385 (2000)

33. I. Perrin, Y. Bidel, N. Zahzam, C. Blanchard, A. Bresson, M. Cadoret, Phys. Rev. A 99, 013601 (2019)

34. S.-w. Chiow, S. Herrmann, S. Chu, H. Müller, Phys. Rev. Lett. 103, 050402 (2009)

35. B. Barrett, P. Cheiney, B. Battelier, F. Napolitano, P. Bouyer, Phys. Rev. Lett. 122, 043604 (2019)

36. D.S. Durfee, Y.K. Shaham, M.A. Kasevich, Phys. Rev. Lett. 97, 240801 (2006)

37. J. Rudolph, Matter-wave optics with Bose-Einstein condensates in microgravity, Doctoral dissertation, Leibniz Universität Hannover, 2016, https ://doi.org/10.15488/ 4702

38. S. Abend, Atom-chip gravimeter with Bose-Einstein condensates, Doctoral dissertation, Leibniz Universität, Hannover, 2017, https://doi.org/10.15488/8921

39. T. van Zoest, N. Gaaloul, Y. Singh, H. Ahlers, W. Herr, S.T. Seidel, W. Ertmer, E.M. Rasel et al., Science 328, $1540(2010)$

40. H. Ahlers, H. Müntinga, A. Wenzlawski, M. Krutzik, G. Tackmann, S. Abend et al., Phys. Rev. Lett. 116, 173601 (2016)

41. R. Geiger, V. Ménoret, G. Stern, N. Zahzam, P. Cheinet et al., Nat. Commun. 2, 474 (2011)

42. D.W. Allan, Proc. IEEE 54, 221 (1966)

43. M. Rudemo, Scand. J. Stat. 9, 65 (1982)

44. G.T. Foster, J.B. Fixler, J.M. McGuirk, M.A. Kasevich, Opt. Lett. 27, 951 (2002)

45. Y.-J. Chen, A. Hansen, G.W. Hoth, E. Ivanov, B. Pelle, J. Kitching, E.A. Donley, Phys. Rev. Appl. 12, 014019 (2019)

46. A.V. Rakholia, H.J. McGuinness, G.W. Biedermann, Phys. Rev. Appl. 2, 054012 (2014)
47. N. Malossi, Q. Bodart, S. Merlet, T. Lévèque, A. Landragin, F. Pereira Dos Santos, Phys. Rev. A 81, 013617 (2010)

48. H. Müller, S.-w. Chiow, Q. Long, S. Herrmann, S. Chu, Phys. Rev. Lett. 100, 180405 (2008)

49. B. Plotkin-Swing, D. Gochnauer, K.E. McAlpine, E.S. Cooper, A.O. Jamison, S. Gupta, Phys. Rev. Lett. 121, 133201 (2018)

50. J.M. McGuirk, M.J. Snadden, M.A. Kasevich, Phys. Rev. Lett. 85, 4498 (2000)

51. G.D. McDonald, C.C.N. Kuhn, S. Bennetts, J.E. Debs, K.S. Hardman, M. Johnsson, J.D. Close, N.P. Robins, Phys. Rev. A 88, 053620 (2013)

52. S.-w. Chiow, T. Kovachy, H.-C. Chien, M.A. Kasevich, Phys. Rev. Lett. 107, 130403 (2011)

53. P. Cladé, S. Guellati-Khélifa, F. Nez, F. Biraben, Phys. Rev. Lett. 102, 240402 (2009)

54. M. Gebbe, S. Abend, J.-N. Siemß, M. Gersemann, H. Ahlers, H. Müntinga, S. Herrmann, N. Gaaloul, C. Schubert, K. Hammerer, C. Lämmerzahl, W. Ertmer, E.M. Rasel, arXiv: 1907.08416 (2019)

55. N. Mielec, M. Altorio, R. Sapam, D. Horville, D. Holleville, L.A. Sidorenkov, A. Landragin, R. Geiger, Appl. Phys. Lett. 113, 161108 (2018)

56. J. Rudolph, W. Herr, C. Grzeschik, T. Sternke, A. Grote, M. Popp, D. Becker, H. Müntinga, H. Ahlers, A. Peters, C. Lämmerzahl, K. Sengstock, N. Gaaloul, W. Ertmer, E.M. Rasel, New J. Phys. 17, 079601 (2015)

57. K.U. Schreiber, T. Klügel, J.-P.R. Wells, R.B. Hurst, and A. Gebauer, Phys. Rev. Lett. 107, 173904 (2011)

58. N. Beverini, A. Di Virgilio, J. Belfi, A. Ortolan, K.U. Schreiber, A. Gebauer, T. Klügel, J. Phys. 723, 12 (2015)

59. D.C. Aveline, J.R. Williams, E.R. Elliott et al., Nature 582, $193(2020)$

60. D. Becker, M.D. Lachmann, S.T. Seidel et al., Nature 562, 391 (2018)

61. K. Frye, S. Abend, W. Bartosch, A. Bawamia, D. Becker, H. Blume et al., arXiv: 1912.04849 (2019) 